\section{A note on the statistical treatment of individual differences in multidimensional scaling}

\author{
DAVID O'HARE \\ University of Exeter, Exeter EX4 4QG, England
}

Multidimensional scaling techniques which retain information about individual differences are being used with increasing frequency in psychology. One of the most popular methods, Carroll and Chang's "INDSCAL" model (1970) has recently been used by Howard and Silverman (1976) to investigate the nature of the psychological dimensions underlying the perception of 16 complex sounds. Choice of the method was apparently influenced by the authors' expectation that "large individual differences would occur in the perception of these sounds" (Howard \& Silverman, 1976, p. 197). Individual differences in the salience of the stimulus dimensions are usually examined by direct comparison of the weightings for each individual on each of the stimulus dimensions, which are computed directly by the INDSCAL procedure. In this case, Howard and Silverman report that, while most subjects relied heavily on the first INDSCAL dimension (corresponding to fundamental frequency), some subjects were found to give equal, or in some cases greater, weighting to the second and third dimensions (corresponding to waveform and number and frequency of formants, respectively). The authors further suggest that these differences can be related to the musical background of their subjects. However, inspection of their INDSCAL subject space where "musical" and "nonmusical" subjects can be compared in terms of their differential weighting of dimensions 1 and 3 described above (Howard \& Silverman, 1976, Figure 7) hardly seems to support the confident assertion that "the difference between musical and nonmusical subjects is obvious in the figure" (Howard \& Silverman, 1976, p. 198). Far from being obvious, the distinction between the two groups appears to be highly tenuous, and any supportive statistical analysis is conspicuously absent. A consideration of Howard and Silverman's data raises an important question concerning the appropriate treatment of individual differences in subject weightings for dimensions obtained from multidimensional scaling analyses. The following comments are therefore offered with specific reference to the conclusions reached by Howard and Silverman, in particular, and to the statistical treatment of subject weightings obtained from the increasingly popular INDSCAL method, in general.
Visual inspection of the subject space, obtained for instance from the INDSCAL procedure, or the application of any kind of linear statistics, are liable to give misleading results for the simple reason that subjects who, in fact, attach equal relative importance to the dimensions are liable to be "pulled apart" by differences in the percentage of variance accounted for in their scalar products data. To avoid this problem, a different approach may be adopted. This is to convert the subject points to angular directionsan elementary trigonometrical procedure-and then apply a body of statistics specially developed for the purpose, known as "directional statistics" (see Mardia, 1972). These are suitable for data in two or three dimensions, and may be found in both parametric and nonparametric forms. Treating Howard and Silverman's data in this way shows that there is, in fact, no difference between the "musical" and "nonmusical" subjects in their relative weighting of the stimulus dimensions.

The steps taken in arriving at this conclusion can be summarized as follows. First, from the subject weightings for dimensions 1 and 3 (since this is where individual differences are claimed to be most evident), each subject's data are converted into the appropriate angle in the two-dimensional subject space. The equivalent descriptive statistics to the linear mean and standard deviation are the mean direction and circular variance. These are computed to be $22^{\circ} 4^{\prime}$ (variance $=0.048$ ) and $30^{\circ} 6^{\prime}$ (variance $=0.036$ ) for the "musical" and "nonmusical" groups, respectively. Alternatively, we could use the weights on all three dimensions and calculate the same statistics based on the sphere instead of the circle. Unfortunately, there is no direct analogue of the $t$ test which can be used to test the significance of differences in mean directions. The most suitable directional test is a parametric test of mean directions described by Mardia (1972, p. 153). This is based on a von Mises distribution, which approximates the normal distribution on the line. The test yields a statistic $\overline{\mathbf{R}}^{\prime}$ which is compared with the expected value. In the present case, the obtained value was 0.03 , which is considerably less than the $5 \%$ critical value of 0.31 . There is, therefore, no significant difference between the mean directions. This is confirmed by the results of the nonparametric equivalent, known as the Uniform-Scores Test (Mardia, 1972, p. 197)., In this test, the angular directions are first converted to equidistant ranks, and a statistic $R^{\prime}$ calculated on the basis of the rankings. This is approximately distributed as chi square. Again, the obtained value of 1.385 falls 
considerably short of the $5 \%$ value of chi square, which is 5.991. Full details of these calculations are of course available from the author.

Howard and Silverman's conclusion that differences in the salience of sound features are a function of previous musical experience is therefore not supported by their data. A failure to disconfirm the null hypothesis in the present study does not, of course, preclude the possibility of doing so in the future. Indeed, in my own research we have found a difference in the salience of features comprising artistic style between those studying visual art and those not (O'Hare, 1976). In general, the utility of individual differences scaling procedures may be enhanced, and the drawing of inappropriate conclusions avoided, by the appropriate application of directional statistics for angular data.

\section{REFERENCES}

Howard, J. H., \& Silverman, F. B. A multidimensional scaling analysis of 16 complex sounds. Perception \& Psychophysics, 1976, 19. 193-200.

MARDIA, K. V. Statistics of directional data. London: Academic Press, 1972.

O'HARE, D. P. A. Individual differences in perceived similarity and preference for visual art: A multidimensional scaling analysis. Perception \& Psychophysics, 1976, 20, 445-452.

(Received for publication November 30, 1976.) 